\title{
An Approach to the Movers' Problem that Combines Oriented Matroid Theory and Algebraic Geometry
}

\author{
Federico Thomas \\ Institut de Cibernètica (CSIC - UPC) \\ Diagonal 647,2 planta \\ 08028 Barcelona, SPAIN \\ e-mail: thomas@ic.upc.es
}

\begin{abstract}
This paper investigates the partition of the configuration space induced by basic contacts between polyhedra, using their combinatorial interpretation in terms of oriented matroids. It is shown that solving motion planning problems using this cellular decomposition can be analytically expressed in terms of invariants, without explicit use of artificial coordinate frames.

One of the main aims of this paper is to draw the attention of the reader to some results from the Theory of Matroids which are directly applicable to path planning, and many other geometric problems arising in Robotics. In particular, the relevance of the concept of mutation in the context of collision-free path planning is highlighted.

Moreover, it is proved that the set of mutations contains the walls of a given cell in configuration space, and that this set, as one moves from one cell to a neighbor one, can be updated in linear time with the number of vertices. This provides a simple way to detect a large amount of redundant constraints from purely combinatorial considerations, without relying on the manipalation of algebraic equations.
\end{abstract}

Keywords: Configuration Space, Movers' problem, Collision-free Path Planning, Oriented Matroids, Chirotopes.

\section{Introduction}

This work deepens on the combinatorial structure of the problem of moving, with six degrees of freedom, a three dimensional simplicial polyhedron between two given locations without colliding with other simplicial polyhedra in the environment ( $f i g .1$ ). This is an instance of the "find-path" or "piano-movers" problem (the reader is addressed to [11] for a textbook on this matter).

The motion planning problem has been studied intensively over the last 15 years and has been found to possess a significant mathematical content. The use of classical geometry, topology, algebraic geometry, algebra, and combinatorics in the analysis of many instances of the problem has attained a high degree of sophistication.

As it was pointed out in [14], because the problem has a rich geometric and combinatorial structure, it is imperative that we understand this structure in detail from a mathematically rigorous point of view and explore it for potential algorithmic improvements.

The papers by Schwartz and Sharir [15, 16] showed the possibility of using algebraic and topological, rather than purely geometric, methods in motion planning. Although, even for a fixed number of d.o.f., the algorithm proposed by them is not useful in practice (no implementation based on this approach has been reported), they have motivated a great deal of research on this direction. This has provided formulations of the path planning problem in terms of semialgebraic set manipulations. Algorithms based on recursive cellular decompositions using the Collins' Cylindrical Algebraic Decomposition [3], such as [16] and its sequels, or on Canny's Roadmap [2] are paradigmatic. The main drawback of these approaches is that they provide a rapid algebraization of the problem thus becoming blind to the underlying geometry. This becomes clear when one realizes that these decompositions are greatly dependent on the location of the reference frame of the moving object, leading in most cases to much more complicated decompositions than those required in practice.

In [4] an algorithm for the movers' problem with six degrees of freedom for polyhedral objects decomposed 

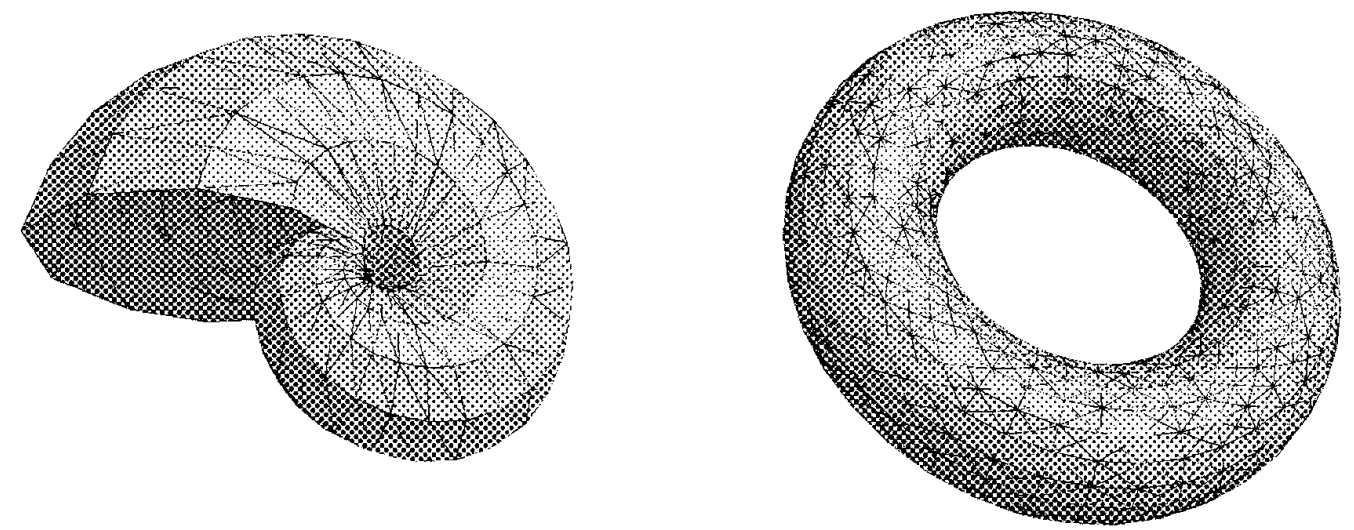

Fig. 1 An instance of our case of the movers' problem: Can the seashell pass through the hole of the torus?

into convex components, that does not generate an explicit representation of the whole configuration space, is designed and implemented. Its main drawback is that it is complete at a level of user-defined resolution, so that arbitrary narrow paths cannot be part of the solution. In Donald's work, the idea of applicability constraint is also introduced. This allowed him to consider a reduced set of constraints at each point of the configuration space. We follow here the same idea: we introduce more constraints than those initially needed, but this allows us to deal with a fairly reduced set of constraints at each point of the configuration space.

This paper also presents the most important results from Matroid Theory that are relevant to Motion Planning. Most of them are given without proof, but the reader is addressed to proper references for them. For readability reasons, results are restricted, or adapted, to the 3-dimensional Euclidean space, thus becoming more intuitive and interesting for practical applications.

The organization of the paper is as follows. Section 2 introduces the idea of oriented matroid associated with two polyhedra, one moving with reference to the other. Section 3 shows that the basic contacts between polyhedra are elements of a matroid. In this section it is also shown that the partition of the CS induced by the basic contacts is not a proper cellular decomposition in the sense that cells are not connected. Section 5 briefly describes the Grassmann-Plücker relations, a fundamental tool in the theory of oriented matroids. Section 4 introduces the concept of mutation and its important role to detect redundant constraints. Finally, conclusions and prospects for future research are described in Section 6 .

\section{Preliminaries}

Let us assume that we have two polyhedra, $P$ and $Q$, with $m$ and $n$ vertices respectively, which are in general position. As a consequence, no face of both polyhedra has more than three vertices. This includes many practical applications in which object surfaces are approximated by triangular meshes. Although extensions, including the general case can be considered, for the moment this is a reasonable simplification that highly easies the treatment that follows.

Now, consider the matrix of all the vertices of both polyhedra:

$$
\mathbf{X}(P, Q):=\left(\begin{array}{cccc}
x_{1}^{1} & x_{2}^{1} & x_{3}^{1} & 1 \\
x_{1}^{2} & x_{2}^{2} & x_{3}^{2} & 1 \\
\vdots & \vdots & \vdots & \vdots \\
x_{1}^{m+n-1} & x_{2}^{m+n-1} & x_{3}^{m+n-1} & 1 \\
x_{1}^{m+n} & x_{2}^{m+n} & x_{3}^{m+n} & 1
\end{array}\right)
$$

where $\mathrm{x}^{i}=\left(x_{1}^{i}, x_{2}^{i}, x_{3}^{i}, 1\right)$ are the homogeneous coordinates of vertex $i$ in $\Re^{3}$, after normalizing the last coordinate to 1 , and let $\Xi$ be a map which assigns to each ordered simplex $\lambda=\left(\lambda_{1}, \lambda_{2}, \lambda_{3}, \lambda_{4}\right)$, with $\lambda_{i} \in$ $\{1, \ldots, n+m\}$ and $\lambda_{1}<\lambda_{2}<\lambda_{3}<\lambda_{4}$, the determinant

$\Xi(\lambda)=\Xi\left(\lambda_{1}, \lambda_{2}, \lambda_{3}, \lambda_{4}\right):=[\lambda]=\left|\begin{array}{llll}x_{1}^{\lambda_{1}} & x_{2}^{\lambda_{1}} & x_{3}^{\lambda_{1}} & 1 \\ x_{1}^{\lambda_{2}} & x_{2}^{\lambda_{2}} & x_{3}^{\lambda_{2}} & 1 \\ x_{1}^{\lambda_{3}} & x_{2}^{\lambda_{3}} & x_{3}^{\lambda_{3}} & 1 \\ x_{1}^{\lambda_{4}} & x_{2}^{\lambda_{4}} & x_{3}^{\lambda_{4}} & 1\end{array}\right|$,

which will be called the oriented volume of the simplex $\lambda$, and let $\chi$ be the map which assigns to each $\lambda$ the sign of $\Xi(\lambda)$; that is, $\chi(\lambda):=\operatorname{sign}([\lambda])$, which will be simply called the orientation of $\lambda$. Note that $\Xi(\lambda)$, and hence $\chi(\lambda)$, are independent of the reference frame.

Let the set of 4-element ordered subsets of $p=n+$ $m$ vertices be identified with the set $\Lambda(p, 4)$ of strictly increasing 4-tuples. That is, 
(a)

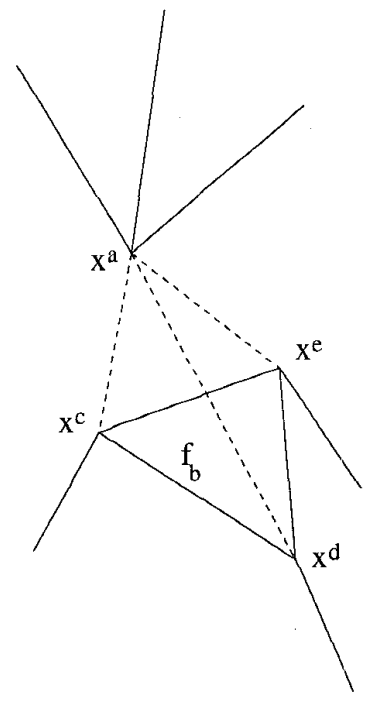

(b)

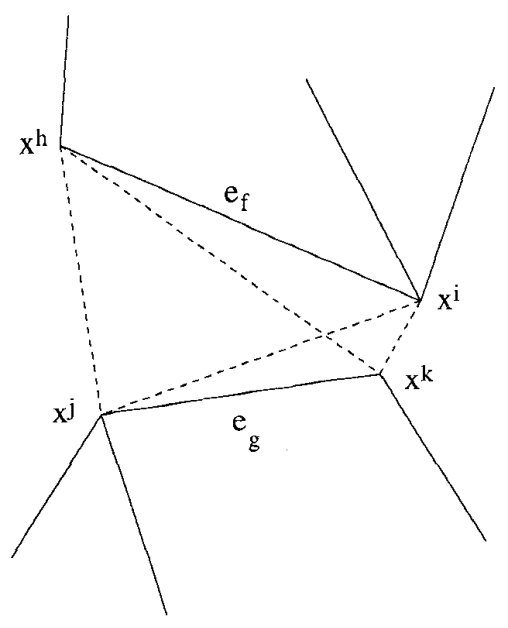

Fig. 2 The two basic contacts and their associated simplices in dotted lines. (a) Type-A; and (b) type-B.

$$
\begin{aligned}
& \Lambda(p, 4):=\left\{\left(\lambda_{1}, \ldots, \lambda_{4}\right) \in\right. \\
& \left.\quad\{1, \ldots, p\}^{4} \mid 1 \leq \lambda_{1}<\ldots<\lambda_{4} \leq p\right\} .
\end{aligned}
$$

Let

$\Xi_{\mathbf{X}}:=([1,2,3,4],[1,2,3,5], \ldots,[p-3, \ldots, p]) \in \Re\left(\begin{array}{c}p \\ 4\end{array}\right)$,

then

$$
\mathcal{G}_{p, 4}^{\Re}:=\left\{\Xi_{\mathbf{X}} \mid \mathbf{X} \in \Re^{4 p}\right\} \subset \Re\left(\begin{array}{c}
p \\
4
\end{array}\right)
$$

is a Grassmann variety (see [10] for a complete description of Grassmanians and other related varieties).

The combinatorial properties of $\chi$ can be studied in the language of oriented matroids, or chirotopes. Actually, $\chi: \Lambda(p, 4) \rightarrow\{-1,1\}$ has the structure of a uniform oriented matroid, or simplicial chirotope, of rank 4 with $p$ points. Oriented matroids play an important role in many parts of Discrete Mathematics. For chirotopes and their relationship with other axiomatics of oriented matroids see [1], a recent source book on the matter.

In our case, there is an important class of simplices: those involving vertices of both polyhedra in which case their orientation depends on the relative location of the polyhedra. This set of simplices will be denoted Moving $(\Lambda(p, 4), n)$.

Here two important points arise:

- When one polyhedron moves continuously with reference to the other, the orientations in Moving $(\cdot)$ define equivalence classes of configurations in which these orientations remain unchanged. This induces a cell decomposition of the configuration space of both polyhedra. This is discussed in Section 3 .

- It is clear that it is not possible to arbitrarily choose the signs of all orientations in Moving( $\cdot)$ and thus it is reasonable to look for a base set of orientations from which all other orientations can be obtained. This is discussed in Section 4.

\section{Constraints, intersection detection and cell decompositions of the configuration space}

Configuration Space (CS) is the product space of the space of translations, $\Re^{3}$, and the space of rotations, $S O(3)$, so that each point, a configuration, in this space represents a relative location between polyhedra $P$ and $Q$.

A configuration, $\mathbf{Y}$, in $\Re^{3} \times S O(3)$ has the form $\mathbf{Y}=$ $(\mathbf{r}, \boldsymbol{\Theta})$, where $\mathbf{r}$ denotes a three-dimensional translation vector, and $\Theta$ some three dimensional rotation.

Now, we are going to introduce the traditional idea of the basic contacts [2]. The reason will become clear shortly.

A type- $A$ contact occurs when a vertex, $x^{a}$, of one polyhedron touches a face, $\mathbf{f}_{b}$, of the other. For this to occur, the vertex must lie in the plane of the face. This can be expressed, assuming $w \log$ that the indices of the generated simplex are already ordered, as 

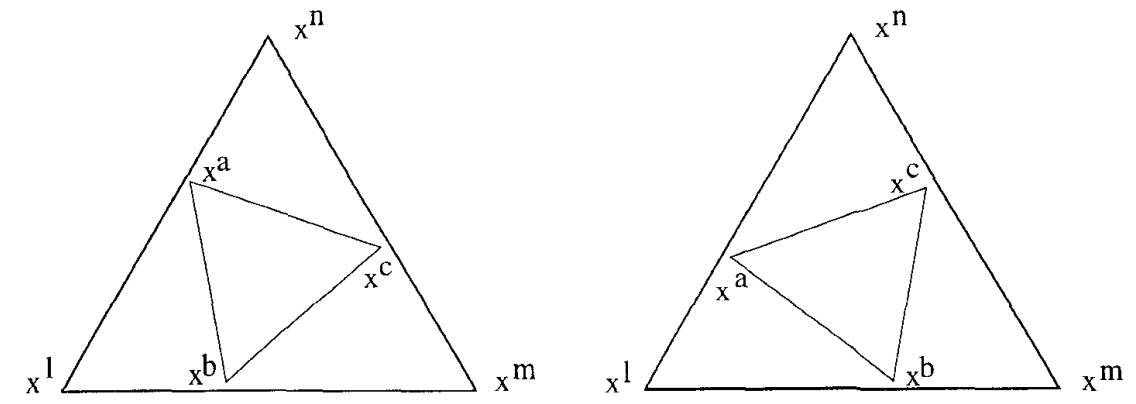

Fig. 3 Two configurations of a moving triangle associated with the same chirotope but located in different cells of the CS.

$$
\Xi(a, c, d, e)=0
$$

where $\mathbf{x}^{c}, \mathbf{x}^{d}$, and $\mathbf{x}^{e}$ are the vertices on face $\mathbf{f}_{b}(f i g .2 a)$.

A type-B contact occurs when an edge, $e_{f}$, from one polyhedron touches an edge, $\mathbf{e}_{g}$, from the other. For this to occur, both edges must lie on the same plane. This can be expressed, as above, as

$$
\Xi(h, i, j, k)=0 \text {, }
$$

where $\mathbf{x}^{h}$ and $\mathbf{x}^{i}$ are the vertices defining $\mathbf{e}_{f}$, and $\mathbf{x}^{j}$ and $\mathbf{x}^{k}$ those defining $\mathbf{e}_{g}(f i g .2 b)$.

Definition 1. (Constraint). The simplices involved in equations (6) and (7) will be called constraints and the set of all constraints will be denoted Constraints $(P, Q)$.

Given a reference frame, the algebraic manifolds represented by equations (6) and (7) in terms of $\mathbf{r}$ and $\Theta$ are called C-surfaces, which induce a partition of configuration space into cells. Actually, each C-surface divides the CS into two half-spaces, each containing all configurations where the orientation of the corresponding simplex has the same sign.

This partition is important because it contains two basic kinds of cells: cells where all configurations in them lead to intersections between the surfaces of both polyhedra and cells free from configurations leading to surface intersections. This means that the decision whether both polyhedra have their surfaces intersecting can be carried out directly from the orientations of the simplices in Constraints $(P, Q)$. This is true even in the case that both polyhedra are not simplicial. The proof is not straightforward and the reader is addressed to [17] for it.

In general, only from the orientations of the simplices in Constraints $(P, Q)$ it is not possible to detect inclusions between $P$ and $Q$. Assuming that we start at a configuration in which both polyhedra are far apart, a configuration in which one is fully inside the other cannot be reached without generating an intersection between their surfaces, and this is enough for most path planning practical applications. Anyway, if objects without interior, such as the seashell in fig. 1, are considered, there is no alternative.

By considering all simplices in Moving $(\Lambda(p, 4), n)$, not only Constraints $(P, Q)$, we can generate a finer partition of the CS so that each simplex in Moving $(\Lambda(p, 4), n)$ has an associated $\mathrm{C}$-surface. In this case, all configurations in each cell of the partition lead to the same orientations of all simplices, i.e. each cell has an associated chirotope. Unfortunately, the converse is not true, that is, a realizable chirotope does not correspond, in general, to a single cell of this partition (a formal definition of realizability will be given in the next section). A simple example of this fact, in the context of $2 \mathrm{D}$ path planning, is shown in fig. 3. While the two configurations shown in it correspond to the same chirotope, i.e. the signs of all involved determinants are identical in both cases, they cannot be moved continuously into each other without violating one of these signs.

In what follows cells refer to the partition induced by $\operatorname{Moving}(\Lambda(p, 4), n)$.

\section{Grassmann-Plücker relations}

For an arbitrary chirotope, the following identities (Grassmann-Plücker relations) are always fulfilled

$$
\begin{aligned}
& \{\lambda \mid \mu\}(\Xi):= \\
& \quad \Xi\left(\lambda_{2}, \lambda_{3}, \lambda_{4}, \lambda_{5}\right) \cdot \Xi\left(\lambda_{1}, \mu_{1}, \mu_{2}, \mu_{3}\right)- \\
& \quad \Xi\left(\lambda_{1}, \lambda_{3}, \lambda_{4}, \lambda_{5}\right) \cdot \Xi\left(\lambda_{2}, \mu_{1}, \mu_{2}, \mu_{3}\right)+ \\
& \Xi\left(\lambda_{1}, \lambda_{2}, \lambda_{4}, \lambda_{5}\right) \cdot \Xi\left(\lambda_{3}, \mu_{1}, \mu_{2}, \mu_{3}\right)- \\
& \\
& \Xi\left(\lambda_{1}, \lambda_{2}, \lambda_{3}, \lambda_{5}\right) \cdot \Xi\left(\lambda_{4}, \mu_{1}, \mu_{2}, \mu_{3}\right)+ \\
& \\
& \quad \Xi\left(\lambda_{1}, \lambda_{2}, \lambda_{3}, \lambda_{4}\right) \cdot \Xi\left(\lambda_{5}, \mu_{1}, \mu_{2}, \mu_{3}\right)=0
\end{aligned}
$$


for $\lambda \in \Lambda(p, 5)$ and $\mu \in \Lambda(p, 3)$.

As a consequence of these relations, the orientations of the simplices involved are not independent.

According to the second fundamental theorem of projective invariant theory, all other relations - such as Laplace extensions - are consequences of these ones. This is also a consequence of the following theorem.

Theorem I. The Grassmann variety $\mathcal{G}_{p, 4}^{\Re}$ is the common zero locus of the corresponding Grassmann-Plücker polynomials. That is,

$$
\begin{array}{r}
\mathcal{G}_{p, 4}^{\Re}=\left\{\quad \Xi \in \Re\left(\begin{array}{c}
p \\
4
\end{array}\right) \mid\{\lambda \mid \mu\}(\Xi)=0,\right. \\
\forall \lambda \in \Lambda(p, 5), \mu \in \Lambda(p, 3)\}
\end{array}
$$

Proof. See [10, p. 65-66].

The following identities

$$
\begin{aligned}
& \{\sigma \mid \tau\}(\Xi):= \\
& \quad \Xi\left(\sigma_{1}, \sigma_{2}, \tau_{1}, \tau_{2}\right) \cdot \Xi\left(\sigma_{1}, \sigma_{2}, \tau_{3}, \tau_{4}\right)- \\
& \quad \Xi\left(\sigma_{1}, \sigma_{2}, \tau_{1}, \tau_{3}\right) \cdot \Xi\left(\sigma_{1}, \sigma_{2}, \tau_{2}, \tau_{4}\right)+ \\
& \quad \Xi\left(\sigma_{1}, \sigma_{2}, \tau_{1}, \tau_{4}\right) \cdot \Xi\left(\sigma_{1}, \sigma_{2}, \tau_{2}, \tau_{3}\right)=0,
\end{aligned}
$$

for $\sigma \in \Lambda(p, 2)$ and $\tau \in \Lambda(p, 4)$, are a particularization of (8), which are known as the three-term GrassmannPlücker relations or simply syzygies, term borrowed from the literature of classical invariant theory. In our case, their relevance comes from the following theorem.

Theorem II. (Las Vergnas). In the uniform case, $\chi(\lambda) \neq 0$ for all $\lambda \in \Lambda(p, 4)$, the three-term GrassmannPlücker relations (10) subsume the five-term relations (8).

Proof. See [6] for a discussion and references where a proof can be found.

Definition 2. (Realizable, coordinalizable or representable chirotope). A chirotope is realizable, or equivalently coordinalizable or representable, iff there exists a set of points in $\Re^{3}$ that generate it.

More formally, a realizable uniform oriented matroid is a point $\chi \in Z_{2}^{\left(\begin{array}{l}p \\ 4\end{array}\right)}$ of the form $\chi=\operatorname{sign}(\Xi)$ for some elements $\Xi \in \mathcal{G}_{p, 4}^{\Re}$ of the corresponding Grassmann variety.

In the identities (10) not all products can have the same sign. Therefore, an unknown orientation might be determined when 5 others are given. This argument can be applied repeatedly to determine all orientations as far as they really are determined (this is discussed in the next section, where the concept of mutation is introduced). If a sign contradiction is found during this inductive process, the chirotope is not realizable. Unfortunately, this is just a necessary condition because we only used orientations instead of oriented volumes. A chirotope leading to no sign contradictions but which is not realizable is called polytopal chirotope.

\section{Mutations, walls and separators}

We will give two characterizations of mutations, the first in terms of Grassmann-Plücker relations, the second in terms of Tucker matrices.

Definition 3. (Mutation, mutant). Let $\chi$ and $\chi^{\prime}$ denote two chirotopes. We will call $\chi$ and $\chi^{\prime}$ mutants if they differ in exactly one simplex orientation, and the simplex will be called a mutation.

This defines in a natural way the mutation graph. $\chi$ and $\chi^{\prime}$ are joined by an edge in this graph if and only if they are mutants.

Proposition I. (Bokowski-Sturmfels). $\lambda \in \Lambda(p)$ is a mutation of $\chi$ if and only if $\chi(\lambda)$ is not determined by any Grassmann-Plücker relation.

In other words, the mutations of $\chi$ are exactly the tuples in $\Lambda(p)$, whose sign is not determined. Let $\operatorname{Mut}(\chi)$ denote this set of simplices.

It can be proved that, if $\chi$ is realizable, then $|\operatorname{Mut}(\chi)| \geq p$, where $p$ is the number of points of $\chi$. This provides another necessary condition for a chirotope to be realizable.

Definition 4. (Reduced System) [8, Def 3.3]. The reduced system of a chirotope $\chi$ on $p$ elements is a subset $\mathcal{R} \in \Lambda(p)$ such that any chirotope $\chi^{\prime}$ on $p$ elements which agrees with $\chi$ on $\mathcal{R}$ is necessarily equal to $\chi$.

The concept of reduced system of a chirotope plays a crucial role in the algorithmical coordinatizability test described in [8, Algorithm 4.2], where an algorithm to obtain a reduced set starting with a set of mutations is also described.

In some cases the mutations are already a reduced system, but in general this cannot be expected.

Remark I. (Roudneff-Sturmfels) [12, Remark 3.2]. Given a chirotope $\chi$, the set $\operatorname{Mut}(\chi)$ is contained in every reduced system $\mathcal{R}$ of $\chi$. 


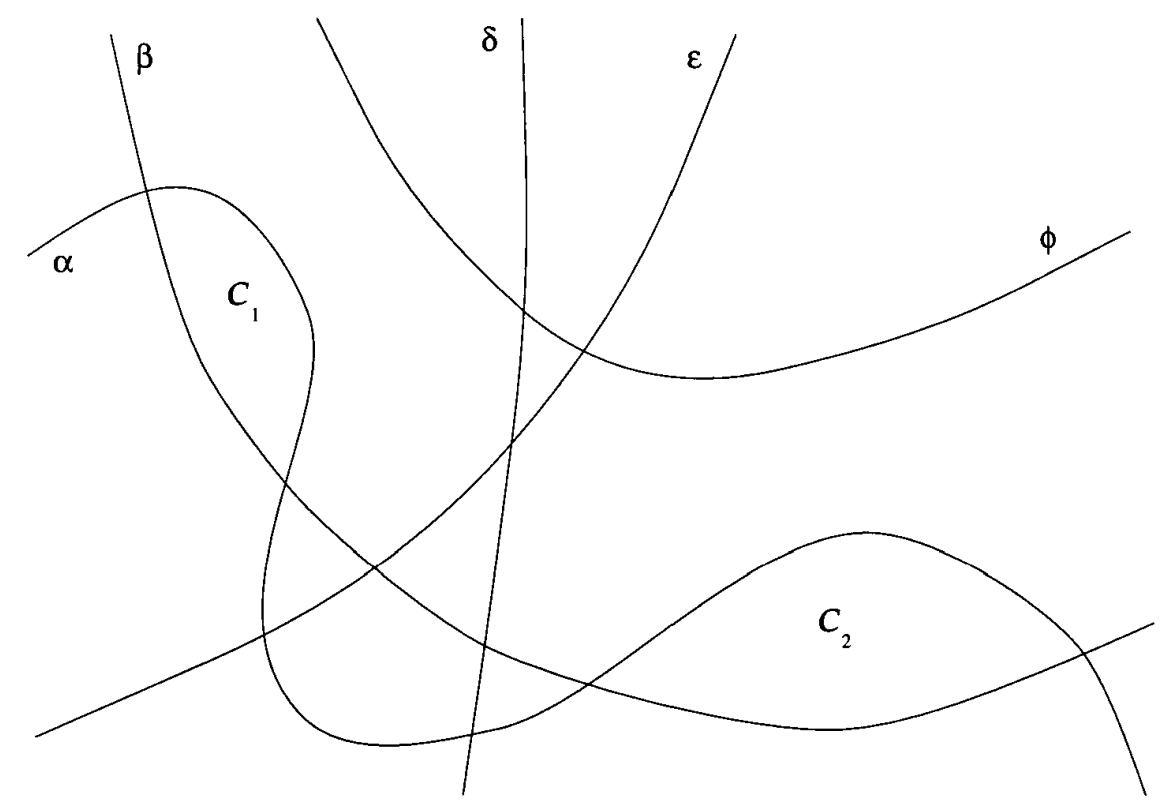

Fig. $4 \Xi(\delta)=0$ and $\Xi(\varepsilon)=0$ act as separators of the two components of the set of configurations define by $\Xi(\alpha)>0$ and $\Xi(\beta)>0$ in $\operatorname{CS}\left(\mathcal{C}_{1} \cup \mathcal{C}_{2}\right)$.

Definition 5. (Tucker Matrix). For $\lambda \in \Lambda(p, 4)$ its associated Tucker matrix, $M_{\lambda}(\Lambda)$, is the following $(p-$ 4) $\times 4$-matrix

$$
M_{\lambda}^{t}(\Lambda):=\left(\begin{array}{ccc}
\ldots & \chi\left(j, \lambda_{2}, \lambda_{3}, \lambda_{4}\right) & \ldots \\
\ldots & \chi\left(\lambda_{1}, j, \lambda_{3}, \lambda_{4}\right) & \ldots \\
\ldots & \chi\left(\lambda_{1}, \lambda_{2}, j, \lambda_{4}\right) & \ldots \\
\ldots & \chi\left(\lambda_{1}, \lambda_{2}, \lambda_{3}, j\right) & \ldots
\end{array}\right)
$$

for $j \in\{1, \ldots, n\} \backslash \lambda$.

Theorem III. (Roudneff-Sturmfels). $\lambda \in \Lambda(p, 4)$ is a mutation of the simplicial chirotope $\chi$ if and only if the Tucker matrix $M_{\lambda}(\Lambda)$ has rank 1 .

Proof. The proof is quite straightforward from the threeterm syzygies (see [12] for details). $\square$

Corollary I. Given a chirotope, the complexity of computing its set of mutations is $O\left(p^{5}\right)$.

Corollary II. Given a chirotope and its set of mutations, if the sign of one of the mutations is changed, the complexity of updating the set of mutations is $O(p)$.

Definition 6. (Walls). Given a configuration Y of CS, those C-surfaces bounding the cell in which it is located are said to correspond to walls of the cell. Walls $(\chi, \mathbf{Y})$ will denote this set.
It is of great relevance to determine the set of walls for a given cell, because they correspond to the smallest set of simplices that are locally relevant to motion planning. In practice, this problem becomes untractable when it is treated from a purely algebraic point of view (see [4]).

Two neighbor cells share a piece of wall of dimension five, so that the configurations in them differ in exactly one simplex orientation: the one associated with this common wall. Thus, two such cells correspond to mutant chirotopes and the common wall, to the mutation. It follows that:

Remark II. The set of walls is contained in the set of mutations, i.e. $\operatorname{Walls}(\chi, \mathbf{Y}) \subset \operatorname{Mut}(\chi)$.

As a consequence, the set $\operatorname{Walls}(\chi, \mathbf{Y})$ is always contained in any reduced system of $\chi$, as expected.

In general, the signs of the simplices in $\operatorname{Walls}(\chi, \mathbf{Y})$ can define more cells than the one in which $\mathbf{Y}$ is included. Taking into account some other simplices, besides those included in the set of walls, some of these extra cells can be separated. These simplices will be called separators (term that we borrow from the CSGbased solid modeling theory [13]). Separators $(\chi)$ will denote this set.

Let us see an example to clarify the above definitions. If the set of configurations $\mathcal{C}_{1}$ (see fig. 4) is the set of points in CS that satisfy 


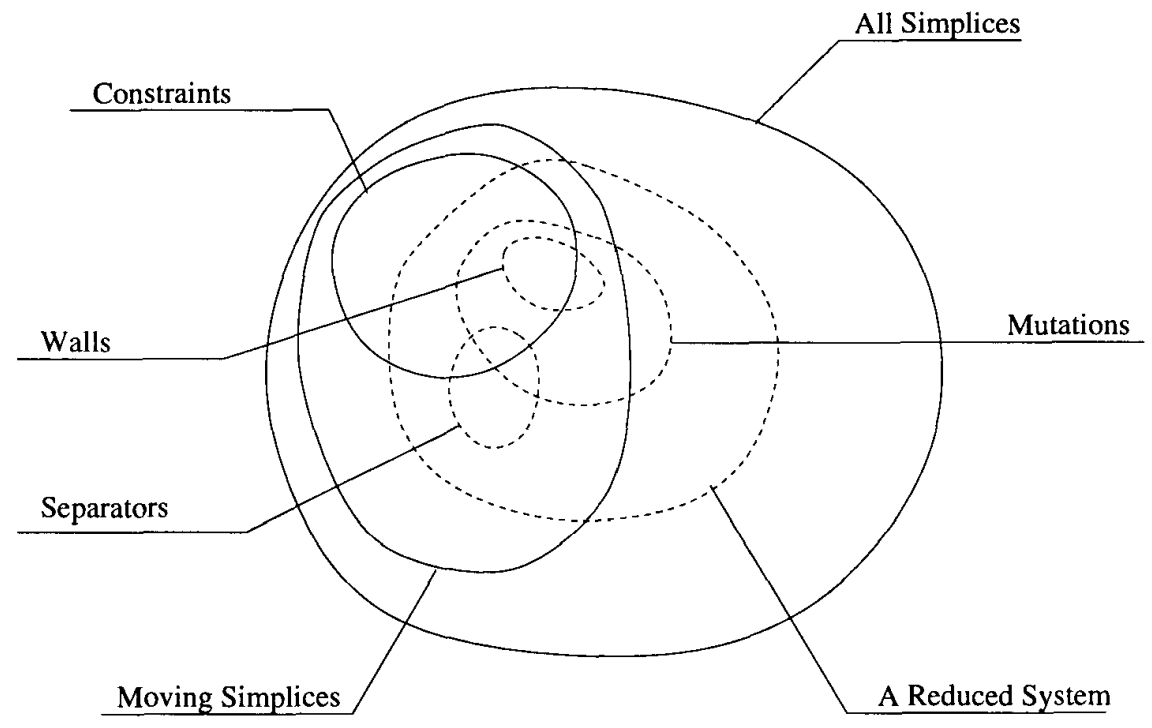

Fig. 5 Classification of simplices. Some of the elements in the sets represented in dotted lines must be reclassified as one moves from one cell of the CS to another one.

$$
\left.\begin{array}{l}
\Xi(\alpha)>0 \\
\Xi(\beta)>0 \\
\Xi(\delta)>0 \\
\Xi(\varepsilon)>0 \\
\Xi(\phi)>0
\end{array}\right\}
$$

then

$$
\left.\left.\begin{array}{l}
\Xi(\alpha)>0 \\
\Xi(\beta)>0 \\
\Xi(\delta)>0
\end{array}\right\} \text { and } \begin{array}{l}
\Xi(\alpha)>0 \\
\\
\Xi(\varepsilon)>0
\end{array}\right\}
$$

are two different reduced systems. $\alpha$ and $\beta$ are mutations. $\delta$ and $\varepsilon$ are separators, and $\phi$ simply corresponds to a redundant orientation.

Note that a mutation might also act as a separator. In the example above, let us suppose that $\delta$ is not contained in our chirotope, then $\varepsilon$ would become a mutation.

The set of mutations is not just composed of walls and possibly separators, it might include other simplices that would correspond to mutations that do not preserve representability. As a consequence, a reduced system is not just composed of walls and separators.

Fig. 5 shows a Venn's diagram of the final classification of simplices, according to the above definitions.

A search in CS, looking for a collision-free path, can be seen as an iterative motion from one cell to a neighbor one traversing one wall. Only when the wall is included in the set of constraints, one has to check for possible intersections. This suggest that an incremental update algorithm should be possible: when a wall is traversed, one imagines detecting which walls expire and which new ones become active. Since this iteration has to be repeated a huge number of times, such an algorithm would be of little interest if its complexity would be high. But, since

$$
\begin{gathered}
\text { Walls }(\chi, \mathbf{Y}) \subset \\
(\operatorname{Mut}(\chi) \cap \operatorname{Moving}(\Lambda(p, 4), n)) \subset \\
\text { Any Reduced System of } \chi
\end{gathered}
$$

we can conclude, according to Corollary II, that a set that tightly contains the walls of a cell can be updated in linear time as one moves from one cell to a neighbor one.

\section{Conclusions}

It has been shown that algebraic geometry approaches to the movers' problem can be benefited from interpreting C-surfaces as elements of a given chirotope. By focusing on the interplay between interference detection based on predicates and the Theory of Oriented Matroids, a new line of research for the movers' problem has been presented.

The most relevant result from Matroid Theory applied here is due to Roudneff and Sturmfels who provided in [12] a linear decision algorithm for the mutation set of a uniform oriented matroid, thus providing a fast and easy 
way to detect a large amount of redundant constraints without solving systems of algebraic equations.

Approaches that are more engineering or AI based and that emphasize approximating, rule-based, or basedcase-tailored solutions, have been implemented but, in general, they are not complete or only complete at a resolution level. An important property of the presented results is that they permit the development of complete algorithms for collision-free path planning, without relying on a user-defined resolution.

Finally, it is worth to mention three points for future research:

- Actually, all results given herein are consequence of the Grassmann-Plücker relations. These relations do not require to consider all simplices, as done here. Thus, the same concepts can be developed for subsets of simplices containing the set of constraints.

- The Grassmann-Plücker relations have a simple geometric interpretation [5]. This interpretation could be extended to mutations. A geometric construction that would permit obtaining the set of mutations would be valuable to attain further complexity improvements.

- The characterization of mutations which preserve realizability is still an open problem, already stated in [12], which deserves further attention to obtain even tighter sets to the set of walls. To this end, a geometric interpretation of mutation would be also very helpful.

\section{Acknowlegments}

I wish to express my thanks to Prof. J. Bokowski for his valuable comments during my stay in Darmstadt, and to $\mathrm{C}$. Torras for fruitful discussions.

Also, financial support by the ESPRIT III Basic Research Actions Program of the EC under contract No. 6546 (project PROMotion) is gratefully acknowledged.

\section{References}

[1] Bjorner, A., Las Vergnas, M., Sturmfels, B., and White, N., Oriented Matroids, Cambridge University Press. 1992.

[2] Canny, J., Complexity of Robot Motion Planning, MIT, Cambridge, Mass., 1987.

[3] Collins, C., "Quantifier Elimination for Real Closed Fields by Cylindrical Algebraic Decomposition," (Proc. 2nd GI Conf. on Automata and Formal Languages). Lecture Notes in Computer Science, Vol. 33, SpringerVerlag, Berlin, pp. 134-163, 1975.
[4] Donald, B.R., Local and Global Techniques for Motion Planning, Master Thesis, MIT, Cambridge, Mass., 1984. A concise version can be found in "A search algorithm for motion planning with six degrees of freedom," Artificial Intelligence, Vol. 31, pp. 295-353, 1987.

[5] Bokowski, J., "On the Geometric Flat Embedding of Abstract Complexes with Symmetries," in Symmetry of Discrete Mathematical Structures and Their Symmetry Groups, K.H. Hofmann and R. Wille (eds.), pp. 1-48, Heldermann Verlag, Berlin, 1990.

[6] Bokowski, J., "Oriented Matroids," in Handbook of Convex Geometry, P.M. Gruber and J.M. Wills (eds.), Elsevier Science Publishers, 1993.

[7] Bokowski, J., Richter, J., and Sturmfels, B., "Nonrealizability Proofs in Computational Geometry," Discrete and Computational Geometry, Vol. 5, pp. 333-350, 1990.

[8] Bokowski, J., and Sturmfels, B., "On the Coordinatization of Oriented Matroids," Discrete and Computational Geometry, Vol. 1, pp. 293-306, 1986.

[9] Bokowski, J., and Sturmfels, B., "Computational Synthetic Geometry," Lecture Notes in Mathematics 1355, Springer, Heidelberg, 1989.

[10] Harris, J., "Algebraic Geometry. A First Course," Graduate Texts in Mathematics 133, Springer, New York, 1992.

[11] Latombe, J-C., Robot Motion Planning, Kluwer Academic Publishers, 1991.

[12] Roudneff, J-P., and Sturmfels, B., "Simplicial Cells in Arrangements and Mutations of Oriented Matroids," Geometria Dedicata, No. 27, pp. 157-170, 1988.

[13] Shapiro, V., and Vossler, D.L. "Separation for Boundary to CSG Conversion," ACM Transactions on Graphics, Vol. 12, No. 1, pp. 35-55, January 1993.

[14] Sharir, M., "Algorithmic Motion Planning in Robotics," IEEE Computer, pp. 9-20, March 1989.

[15] Shwartz, J.T., and Sharir, M., "On the Piano Movers" Problem: I," Comm. Pure and Applied Math., Vol. 36, pp. 345-398, 1983.

[16] Shwartz, J.T., and Sharir, M., "On the Piano Movers" Problem: II. General Techniques for Computing Topological Properties of Real Algebraic Manifolds," Adv. Appl. Math., Vol. 4, pp. 298-351, 1983.

[17] Thomas, F., and Torras, C., "Interference Detection Between Non-Convex Polyhedra Revisited with a Practical Aim," IEEE Int. Conf. on Robotics and Automation, pp. 587-595, May 1994. 